\title{
Efecto del Salvado de Trigo en las Propiedades Fisicoquímicas y Sensoriales del Yogurt de Leche de Búfala
}

\author{
Mónica M. Simanca, Ricardo D. Andrade y Margarita R. Arteaga \\ Facultad de Ingenierías, Departamento de Ingeniería de alimentos, Universidad de Córdoba, \\ Km 12 Vía Cereté-Ciénaga de Oro, Colombia (e-mail: msimanca@correo.unicordoba.edu.co)
}

Recibido Ago. 01, 2012; Aceptado Sep. 28, 2012; Versión final recibida Oct. 06, 2012

\begin{abstract}
Resumen
Se evaluó el efecto de la adición de salvado de trigo (en concentraciones $0,1,3$ y $5 \% \mathrm{p} / \mathrm{v}$ ) y el tiempo de almacenamiento en las propiedades fisicoquímicas y sensoriales del yogurt de leche de búfala. Al producto obtenido se le realizaron pruebas fisicoquímicas (acidez, $\mathrm{pH}$, materia grasa, sólidos totales, sinéresis y densidad) y análisis de aceptación sensorial utilizando una escala hedónica de 9 puntos con 50 catadores. La adición de salvado de trigo influyó de manera directamente proporcional sobre la acidez, sinéresis, sólidos totales y densidad, e inversamente sobre el $\mathrm{pH}$ y la aceptación sensorial del producto. Durante el tiempo de almacenamiento se observó un aumento de la acidez y sinéresis, lo cual evidenció pérdida de calidad del producto. El análisis sensorial mostró que el yogurt de leche de búfala con $1 \%$ de adición de salvado de trigo presenta características organolépticas similares al yogurt con leche de búfala sin adición de fibra.
\end{abstract}

Palabras clave: yogurt, leche de búfala, salvado de trigo, análisis sensorial, producto lácteo

\section{Effect of Bran Wheat on the Physico-Chemical and Sensory Properties of Buffalo Milk Yoghurt}

\begin{abstract}
The effect of adding wheat bran (at concentrations of $0,1,3$ and $5 \% \mathrm{w} / \mathrm{v}$ ) and of the storage time on physicochemical and sensory properties of buffalo milk yoghurt were evaluated. Physicochemical tests, such as acidity, $\mathrm{pH}$, fat, total solids, syneresis and density were done and sensory acceptance analysis using the 9point hedonic scale with 50 tasters were performed. The addition of wheat bran directly influenced acidity, syneresis, total solids and density, and inversely influenced $\mathrm{pH}$ and sensory acceptance of the product. During the storage period there was an increase in acidity and syneresis, which showed loss of product quality. Sensory analyses showed that buffalo milk yoghurt with $1 \%$ of wheat bran presented organoleptic characteristics similar to that of yoghurt without bran addition.
\end{abstract}




\section{INTRODUCCIÓN}

La producción mundial de leche de búfala estimada para el 2009 fue de 89 millones de toneladas, lo que representa un aumento promedio desde 1998 del 3,5\% anual y contribuye al 13\% de la producción mundial de leche, ocupando el segundo lugar después de la leche de vaca, la cual presenta una producción anual de 580 millones de toneladas (IDF, 2009). Las características fisicoquímicas y organolépticas de la leche de búfala facilitan su identificación: presenta un sabor peculiar, levemente endulzado y color blanco, debido a la ausencia casi total de carotenos en su grasa (Andrade et al., 2010; Patiño, 2004). Cada vez es mayor el interés en investigaciones e inversión por la leche de búfalo en varios países, debido principalmente a su atractivo contenido de nutrientes. En comparación con la leche de vaca, la leche de búfala tiene un mayor contenido de grasa, proteína cruda, lactosa, sólidos totales, vitaminas y minerales, que la convierten en un ingrediente adecuado para la fabricación de una amplia variedad de productos lácteos, tales como queso, mantequilla, helados y yogurt (Ahmad et al., 2008; Andrade et al., 2009; Hussain et al., 2012).

El yogurt es un derivado lácteo obtenido por fermentación controlada de la leche con cultivos de bacterias ácido lácticas, las cuales provocan un sabor y aroma típico. Su consumo ha aumentado en los últimos años, considerándose un alimento saludable y de fácil acceso para los consumidores; que tiene entre otros atributos la reducción de los síntomas de intolerancia a la lactosa, ser una fuente importante para la absorción de calcio a través de los cultivos presentes e incremento de la flora microbiana del tracto intestinal (Diaz et al., 2004; Mckinley, 2005). La incorporación de nuevos ingredientes como fibra, vitaminas, y calcio entre otros nutrientes, en la formulación del yogurt, cambia la estructura física y química original del gel, convirtiéndolo en un alimento funcional, por lo que es importante conocer sus efectos. Al yogurt de leche de bufala se le ha adicionado probioticos, evaluando su viabilidad (Shoji et al., 2012; Espírito-Santo et al., 2012). Ademas, el aumento del contenido de fibra en el yogurt, disminuye la sinéresis y la aceptación por parte de los catadores, pero no tiene consecuencias en la variable acidez y $\mathrm{pH}$; por otra parte durante el almacenamiento se presenta pérdida de valor de estos atributos de calidad, lo que disminuye su vida de anaquel (Diaz et al., 2004; Saxelin et al., 2003). En el presente trabajo se analizó el efecto de la adición de salvado de trigo como fuente de fibra, en las propiedades fisicoquímicas y sensoriales del yogurt batido a base de leche de búfala.

\section{MATERIALES Y MÉTODOS}

Recolección y preparación de la materia prima.

La leche de búfala proveniente de la hacienda La fortaleza (Córdoba, Colombia), se transportó en cantinas de aluminio, previamente lavadas y desinfectadas, hasta el taller de lácteos de la planta piloto de la Universidad de Córdoba (Sede Berástegui). Para cada tratamiento se utilizaron 5 litros de leche, previamente filtradas y estandarizadas en un $2,8 \%$ de grasa.

\section{Elaboración del yogurt.}

En la figura 1 se presenta el proceso de elaboración del yogurt batido de acuerdo a la metodología descrita por Tamine y Robinson (1991), pero estandarizando la leche a un $2,8 \%$ de grasa y sin aumento del extracto seco, debido que para mejorar la consistencia y textura del yogurt a partir de leche de búfala no es necesario la adición de leche en polvo (Tamime y Robinson, 1991; Verruma y Salgado, 1994).

Se utilizó un cultivo liofilizado de Lactobacillus delbrueckii spp. bulgaricus y Streptococcus salivarius spp. Thermophilus; como fuente de fibra se utilizó salvado de trigo, obtenido de los supermercados de la ciudad de Montería; reduciéndole el tamaño hasta un diámetro de $50 \mu \mathrm{m}$, utilizando un martillo de cizalla. La adición de la fibra se realizó durante el rompimiento del gel.Se aplicaron cuatro tratamientos (T1, T2, T3 y T4) que consistieron en la adición de 0,1, 2 y $3 \%$ p/v de salvado de trigo, respectivamente.

\section{Evaluación del producto final.}

Al yogurt se le realizaron análisis fisicoquímicos y sensoriales durante los días $0,7,14$ y 21 de almacenamiento. Los análisis fisicoquímicos realizados fueron: acidez por el método titrimétrico de la AOAC 947.05 (AOAC, 2005), pH por valoración potenciométrica AOAC 973.41 (AOAC, 2005), sinerésis por el método de centrifugación propuesto por Díaz et al. (2004), materia grasa por el método de Gerber AOAC 15.030 (AOAC, 2005), sólidos totales por el método de estufa modificado AOAC 925.23 (AOAC, 2005), y densidad por el método gravimétrico AOAC 15.015 (AOAC, 2005). Para la evaluación sensorial del producto final se aplicaron pruebas de aceptación, utilizando una escala hedónica de nueve puntos con 50 catadores escogidos al azar. 


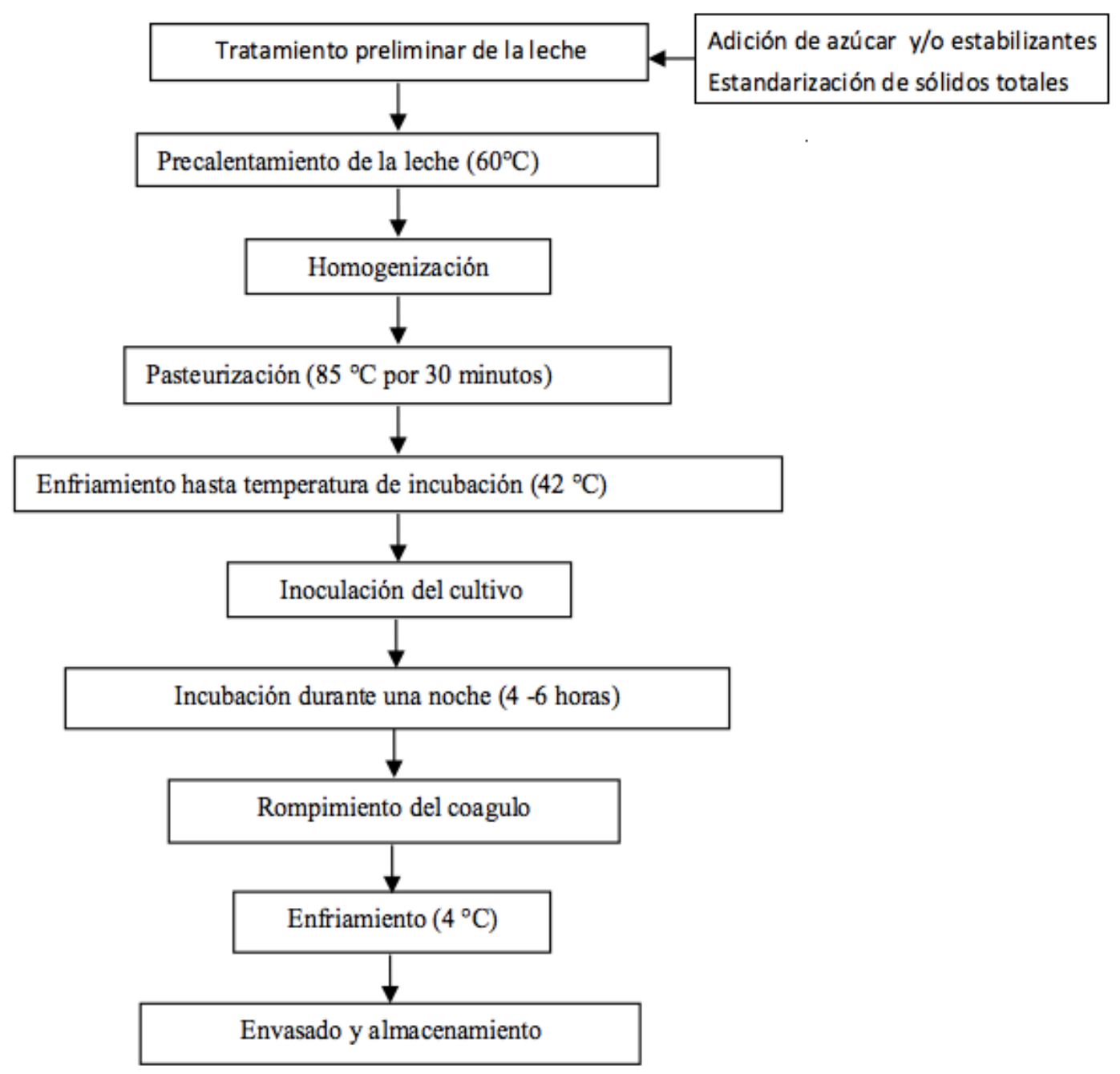

Fig. 1: Flujograma del proceso de elaboración del yogurt entero batido.

\section{Análisis Estadístico.}

Se dispuso de un diseño completamente al azar, bajo una estructura de tratamiento simple con cuatro tratamientos correspondientes a las concentraciones de salvado de trigo adicionado al yogurt de leche de búfala de $0,1,2$ y $3 \%$. Los tratamientos se evaluaron durante los $0,7,14$ y 21 días de almacenamiento, realizando tres repeticiones. Los resultados se analizaron estadísticamente por análisis de varianza (ANOVA) y fue aplicado un test de comparación de Tukey $(p<0,05)$, utilizando el software STATISTICA versión 6.0 .

Para el análisis sensorial se analizaron los tratamientos mediante un diseño en bloque conformado por los catadores, con el fin de observar si existen diferencias en la calificación obtenida por cada uno de los tratamientos, de ser así se determinaron el o los tratamientos en donde se obtengan los mejores porcentajes de aceptación.

\section{RESULTADOS Y DISCUSIÓN}

\section{Características fisicoquímicas del yogurt}

En la tabla 1 se observan los valores promedio de las características fisicoquímicas de los diferentes tratamientos de yogurt. De acuerdo al análisis de varianza se apreciaron diferencias significativas $(p<0,05)$ en los valores de acidez y $\mathrm{pH}$ entre tratamientos y tiempo de almacenamiento. Los valores de acidez del yogurt de leche de búfala con adición de fibra son superiores a los reportados por Díaz et al. (2004) para 
yogurt de leche vaca con adición de fibra, pero concuerdan con los reportados por Cunha et al. (2005) y Scholz y Antunes (1996) para yogurt a partir de leche de búfala. La adición de salvado de trigo incrementa la acidez, esto se debe a que la fibra puede aporta ácidos que hacen que incrementa la acidez y disminuya el $\mathrm{pH}$. Los valores de $\mathrm{pH}$ están dentro del rango admisible y concuerdan con los resultados obtenidos para yogurt con leche de búfala (Cunha et al., 2005) y yogurt con leche de vaca y adición de linaza (Hernández, 2004). Ademas, el pH del yogurt de leche de búfala durante los días de almacenamiento fue mayor que 4 , lo que concuerda con lo reportado por Díaz et al. (2004).

Tabla 1. Características fisicoquímicas del yogurt de leche de búfala (*Letras diferentes indican diferencias significativas).

\begin{tabular}{|c|c|c|c|c|c|c|}
\hline $\begin{array}{c}\text { Salvado de trigo } \\
(\%)\end{array}$ & $\begin{array}{c}\text { Acidez } \\
(\% \mathrm{AL})\end{array}$ & $\mathrm{pH}$ & $\begin{array}{c}\text { Sinéresis } \\
(\%)\end{array}$ & $\begin{array}{c}\text { Materia grasa } \\
(\% \mathrm{p} / \mathrm{v})\end{array}$ & $\begin{array}{c}\text { Sólidos totales } \\
(\% \mathrm{p} / \mathrm{p})\end{array}$ & $\begin{array}{c}\text { Densidad } \\
\left(\mathrm{kg} / \mathrm{m}^{3}\right)\end{array}$ \\
\hline $0(\mathrm{~T} 1)$ & $1,082 \pm 0,25^{\mathrm{a}}$ & $4,412 \pm 0,10^{\mathrm{a}}$ & $43,810 \pm 3,1^{\mathrm{a}}$ & $2,821 \pm 0,07^{\mathrm{a}}$ & $13,373 \pm 0,011^{\mathrm{a}}$ & $1068 \pm 1,5^{\mathrm{a}}$ \\
\hline $1(\mathrm{~T} 2)$ & $1,164 \pm 0,21^{\mathrm{b}}$ & $4,375 \pm 0,11^{\mathrm{b}}$ & $47,472 \pm 3,6^{\mathrm{b}}$ & $2,775 \pm 0,02^{\mathrm{ab}}$ & $14,066 \pm 0,008^{\mathrm{b}}$ & $1081 \pm 1,5^{\mathrm{b}}$ \\
\hline $2(\mathrm{~T} 3)$ & $1,214 \pm 0,20^{\mathrm{c}}$ & $4,343 \pm 0,12^{\mathrm{c}}$ & $51,648 \pm 6,7^{\mathrm{c}}$ & $2,725 \pm 0,01^{\mathrm{bc}}$ & $14,863 \pm 0,003^{\mathrm{c}}$ & $1095 \pm 2,1^{\mathrm{c}}$ \\
\hline $3(\mathrm{~T} 4)$ & $1,283 \pm 0,23^{\mathrm{d}}$ & $4,300 \pm 0,11^{\mathrm{d}}$ & $52,359 \pm 3,4^{\mathrm{c}}$ & $2,688 \pm 0,08^{\mathrm{c}}$ & $15,572 \pm 0,004^{\mathrm{d}}$ & $1115 \pm 2,0^{\mathrm{d}}$ \\
\hline
\end{tabular}

En las figura 2 se aprecia un incremento en la acidez y un decrecimiento en el pH en todos los tratamientos a lo largo del período de almacenamiento, este comportamiento se debe a la actividad del cultivo láctico; similar comportamiento se ha obtenido en investigaciones realizadas en yogurt a partir de leche de vaca (Pirkul et al., 1997) y búfala (Cunha et al. 2005) y en yogurt con diferentes aditivos con leche de vaca (Mehmood et al., 2008). Los cambios en la acidez y pH a través del tiempo de almacenamiento se presentan por las transformaciones bioquímicas que se dan en el yogurt y estas obedecen en gran parte a la acción del cultivo láctico agregado que produce acido láctico a partir de la lactosa.

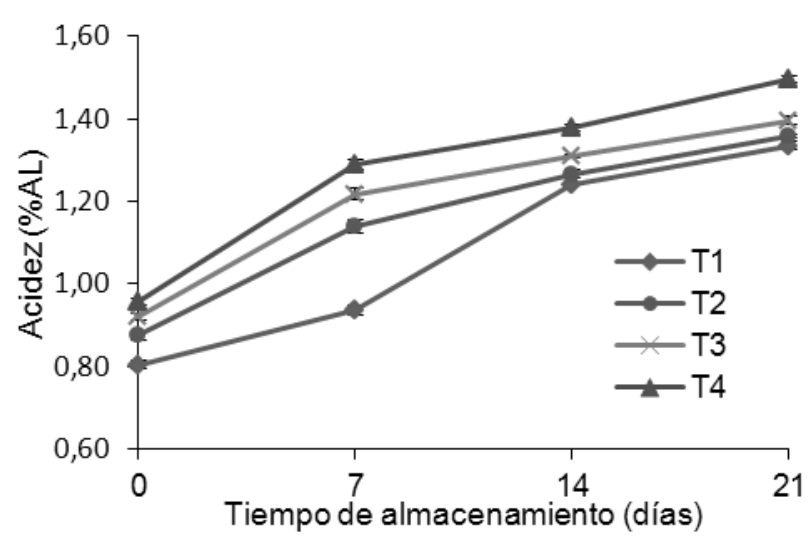

A

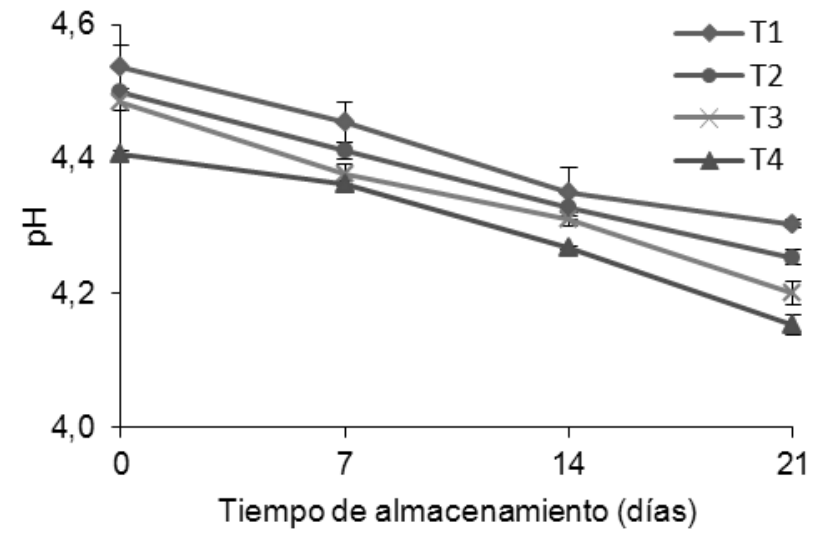

B

Fig. 2: Evolución de la acidez $(\mathrm{A})$ y pH (B) del yogurt de leche de búfala durante el almacenamiento

Según el ANOVA existen diferencias significativas entre los valores promedio de la sinéresis entre tratamientos y durante el almacenamiento. A medida que aumenta la concentración de salvado de trigo adicionado al yogurt aumenta la sinéresis, este resultado no concuerda con lo obtenido por Díaz et al. (2004); lo cual puede ser debido a la diferencia de leches utilizadas, ya que la leche de búfala contiene mayor contenido de sólidos totales, acidez y pH comparada con la leche de vaca. La estructura de los geles de la leche fermentada, se ve influencia con el $\mathrm{pH}$, calor, tipo de cultivo empleado y principalmente la composición de la leche (Leyes y Marshall, 2001).

El grado de sinéresis de los tratamientos observado en la figura 3 se debe a la pérdida de estabilidad y de retención de agua de la los componentes del yogurt, además de las modificaciones estructurales que presenta el gel formado, lo que implica pérdida de agua; otros factores que causas la sinéresis, son la temperatura de incubación heterogénea o excesiva, así como enfriamiento insuficiente durante el proceso (Vélez y Rivas, 2001). Según Tamime y Robinson (1991), la estructura del gel no es adecuada cuando el porcentaje de sinéresis es mayor al $42 \%$; cabe aclarar que este parámetro es para yogurt sin adición de fibra. Solamente el yogurt de leche de búfala sin adición de fibra hasta el día 7 de almacenamiento cumple con lo anterior, esto posiblemente se debe a una deficiente homogenización de la leche, que se refleja en el 
aumento de la sinéresis con el tiempo; este comportamiento también lo presentó el yogurt de leche de vaca con adición de fibra. Respecto a los tratamientos T2, T3 y T4 que presentan porcentajes de sinéresis mayores de $42 \%$ durante todo el almacenamiento, los cuales aumentan con el incremento en la adición de fibra, se deben a la ineficiente homogenización y a la adición de fibra; resultados concordante con los expuestos por Hernández (2004).

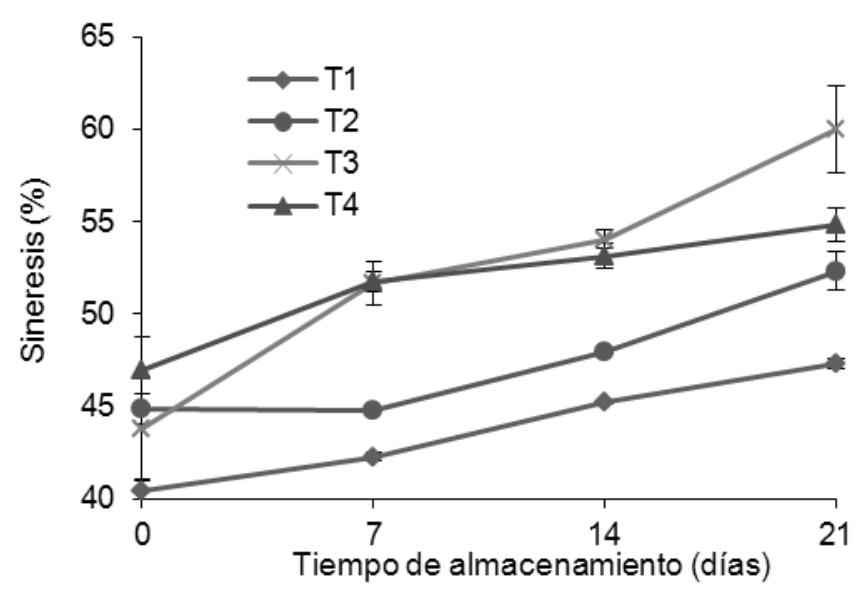

Fig. 3: Evolución de la sinéresis (\%) del yogurt de leche de búfala durante el almacenamiento

El análisis de varianza reveló que existen diferencias significativas en el contenido de grasa y sólidos totales entre los tratamientos. El contenido de grasa de los tratamientos disminuye con el aumento del salvado de trigo, y se debe a la estandarización realizada a la leche de búfala utilizada como materia prima, como lo ratifican Verruma et al. (1994), al concluir que los valores de grasa, sólidos totales y ceniza del yogurt elaborado con la leche de búfala, están directamente relacionado con la composición inicial de la leche.

No se encontraron diferencias significativas durante el tiempo de evaluación para el contenido de grasa y de sólidos totales de los tratamientos (Ver figura 4). Estos mismos resultados fueron observados por Cunha et al. (2005), al evaluar la influencia de diferente contenido de grasa en las características fisicoquímicas y sensoriales del yogurt a partir de leche de búfala. El resultado se debe a que durante el almacenamiento no hay variaciones en el contenido de grasa y de sólidos totales ya que esta se establece según la estandarización de la leche utilizada como materia prima, el contenido de fibra adicionado no interfiere en el porcentaje de grasa pero si en el contenido de sólidos totales.

En la figura 5 se muestra que los valores de densidad obtenidos en los tratamientos evaluados estuvieron entre 1060 y $1120 \mathrm{~kg} / \mathrm{m} 3$, estos valores son inferiores a los reportados para yogures de leche de vaca con salvado de trigo, debiéndose estas variaciones principalmente al porcentaje de adición utilizadas en el estudio de Díaz et al. (2004). Se encontraron diferencias significativas entre los tratamientos; a medida que aumenta el porcentaje de salvado de trigo aumenta la densidad, esto se debe a que la adición de fibra, incrementa los sólidos en el yogurt y por lo tanto aumenta la densidad. No se encontró diferencia significativa entre los tiempos evaluados de los tratamientos, esto concuerda con lo reportado para yogurt de leche de vaca con adición de fibra (Cunha et al., 2005).

\section{Evaluación sensorial del yogurt}

En el análisis sensorial de los productos, realizado a través de la prueba de aceptación con los 50 catadores, se seleccionaron 40 con base en el grado de dispersión de las respuestas del atributo sensorial. En el ANOVA se encontraron diferencias significativas en la percepción de los jueces en el contenido de salvado de trigo y tiempo de almacenamiento. En la tabla 2 se presentan los valores promedios de la calificación sensorial, donde se observa que entre los tratamientos a los que se les incorporó salvado de trigo, el mejor calificado fue el de menor adición (1\%). Resultados similares se encontraron en el yogurt de leche de vaca adicionado con fibra, reportado por Diaz et al., 2004. Así mismo, se observa que el tratamiento con menor adición de fibra es el más parecido al tratamiento sin adición de salvado, hecho que sugiere la necesidad de enmascarar el sabor proporcionado por la fibra mediante un saborizante. Además, se observó que el puntaje promedio de los catadores aumentaba con el tiempo de almacenamiento del yogurt, lo cual puede ser atribuido al aumento de la acidez que enmascararía el sabor a salvado de trigo. 


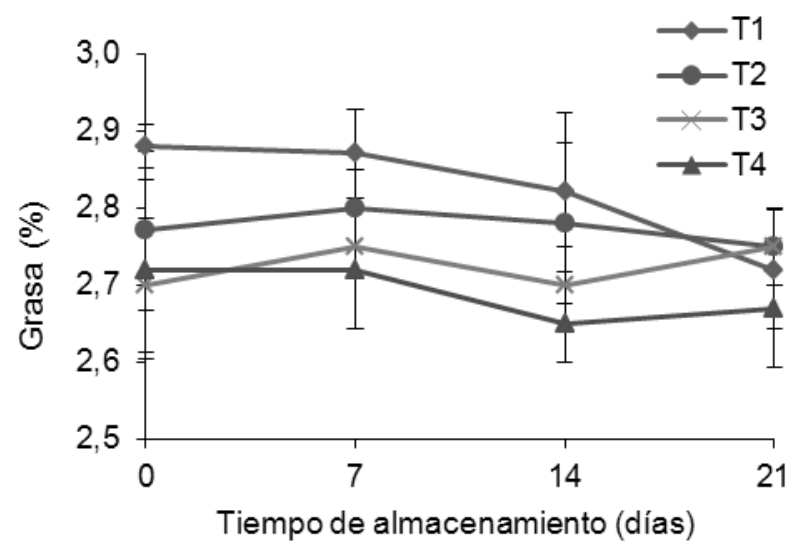

A

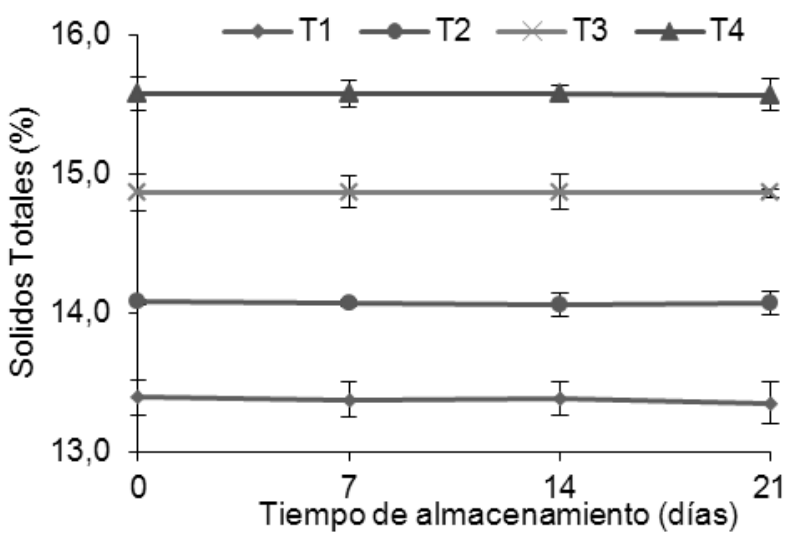

B

Fig. 4: Evolución del contenido de la materia grasa $(A)$ y solidos totales $(B)$ del yogurt de leche de búfala durante el almacenamiento

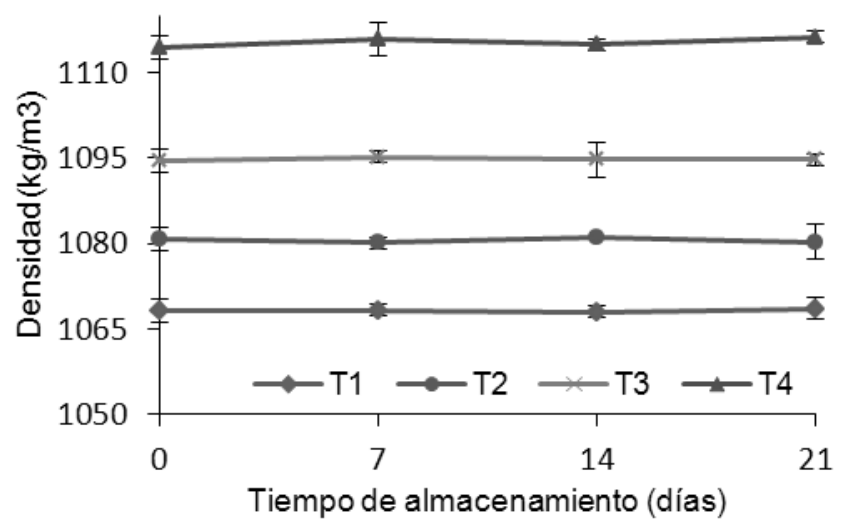

Fig. 5: Evolución de la densidad del yogurt de leche de búfala durante el almacenamiento

Tabla 2: Calificaciones promedio del panel de catadores para yogurt (*Letras diferentes indican diferencias significativas).

\begin{tabular}{|c|c|c|c|}
\hline Tratamiento & Puntaje & Tiempo (días) & Puntaje \\
\hline 1 & $7,554^{\mathrm{a}}$ & 0 & $5,302^{\mathrm{a}}$ \\
\hline 2 & $6,173^{\mathrm{b}}$ & 7 & $5,650^{\mathrm{b}}$ \\
\hline 3 & $4,442^{\mathrm{c}}$ & 14 & $5,669^{\mathrm{c}}$ \\
\hline 4 & $4,169^{\mathrm{d}}$ & 21 & $5,717^{\mathrm{d}}$ \\
\hline
\end{tabular}

Los resultados sensoriales de los tratamientos son consistentes con los arrojados en el análisis fisicoquímico, ya que los tratamientos mejor evaluados y con menor porcentaje de fibra, presentaron mayor $\mathrm{pH}$ y menores valores de acidez y sinéresis. Durante el tiempo se observó un aumento del grado de aceptación del yogurt por parte de los catadores, resultados que no concuerdan con los obtenidos por Atta et al. (2009) en yogurt de leche de búfala con diferentes concentraciones de cultivo iniciadores, y con los obtenidos en el análisis fisicoquímico, ya que los tratamientos con menor adición de fibra presentaron mayor $\mathrm{pH}$ y menores valores de acidez y sinéresis; pero son similares a los obtenidos en el aspecto sensorial en el yogurt natural elaborado con leche de búfala durante el tiempo de almacenamiento (Cunha et al., 2005). Este hecho puede atribuirse al efecto que ejerce en los catadores la acidez, la cual enmascara el sabor del salvado de trigo, influyendo en las mejores calificaciones a medida que aumenta el tiempo de almacenamiento. 


\section{CONCLUSIONES}

La adición de hasta un 3\% de salvado de trigo a yogurt de leche de búfala incrementa la acidez, sinéresis, sólidos totales y densidad, mientras que disminuye su $\mathrm{pH}$ y contenido de materia grasa. A medida que transcurrió el tiempo de almacenamiento aumentó la acidez, sinéresis, y disminuyó el pH del yogurt de leche de búfala; mientras que el contenido de materia grasa, sólidos totales y densidad, no se vio afectado durante el almacenamiento.

Desde el punto de vista sensorial, es posible elaborar yogurt con leche de búfala con 1\% de adición de salvado de trigo, puesto que este producto presenta características organolépticas similares al yogurt con leche de búfala sin adición de fibra, destacándose una mayor aceptación a medida que transcurren los días de almacenamiento.

\section{AGRADECIMIENTOS}

Los autores agradecen a la Universidad de Córdoba, Colombia y a la Hacienda La Fortaleza (Don Eduardo Roldán), por su apoyo en la realización de esta investigación.

\section{REFERENCIAS}

Ahmad, S., y otros 6 autores, Effects of acidification on physico-chemical characteristics of buffalo milk: a comparison with cow's milk, Food Chemistry, 106 (1), 11-17 (2008).

Andrade, R., M. Arteaga y M. Simanca, Efecto del salvado de trigo en el comportamiento reológico del yogurt de leche de búfala, Información Tecnologica, 21(5), 117-124 (2010).

Andrade, R., G. Vélez, M. Arteaga, Y. Díaz y S. Sánchez, Efecto de la neutralización y adición de edulcorante en las propiedades fisicoquímicas, microbiológicas y sensoriales del arequipe de leche de búfala, Vitae, 16 (2), 201-209 (2009).

AOAC, Association of Official Analytical Chemistry. Method of Analysis. 18th edition, 1792. Station, Washington DC. (2005).

Atta, M., M. Hashim, A. Zia y T. Masud, Influence of different amounts of starter cultures on the quality of yoghurt prepared from buffalo milk, Pakistan Journal of Zoology, 41 (9), 129-134 (2009).

Cunha, O., C. Oliveira, R. Hotta y P. Sobral, Avaliação físico-química e sensorial do iogurte natural produzido com leite de búfala contendo diferentes níveis de gordura, Ciência e Tecnologia de Alimentos, 25(3), 448-453 (2005).

Diaz, B., M. Sosa y J. Vélez, Efecto de la adición de fibra y la disminución de grasa en las propiedades fisicoquímicas del yogur, Revista mexicana de ingeniería química, 3(3), 287-305 (2004).

Espírito-Santo, A.P., P. Perego, A. Converti y M.N. Oliveira, Influence of milk type and addition of passion fruit peel powder on fermentation kinetics, texture profile and bacterial viability in probiotic yoghurts, LWTFood Science and Technology, 47(2), 393-399 (2012).

Hernández, P., Evaluación de las propiedades fisicoquímicas y reológicas de yogurt bajo en grasa enriquecido con fibra y calcio de yogurt, Tesis de Maestría en Ciencia de Alimentos, Universidad de las Américas, Puebla, México (2004).

Hussain, I., J. Yan, A.S. Grandison y A.E. Bell, Effects of gelation temperature on Mozzarella-type curd made from buffalo and cows' milk: 2. Curd yield, overall quality and casein fractions, Food Chemistry, 135(3), 1404-1410 (2012).

IDF. International Dairy Federation, The world dairy situation 2009. Bulletin No.438/2009.

Leyes, A. y V. Marshall, The relevance of exopolysaccharides to the rheological properties in milk fermented with ropy strains of lactic acid bacteria, International Dairy Journal, 11(9), 709-721 (2001).

Mckinley, M., The nutrition and health benefits of yogurt, International Journal of Dairy Technology, 58 (1), 112 (2005). 
Mehmood, S., T. Masud, T. Mahmood y S. Maqsud, Effect of different additives from local source on the quality of yogurt, Pakistan Journal of Nutrition, 7(5), 695-699 (2008).

Patiño, E., Factores que afectan las propiedades físicas y la composición química de la leche de búfalas (Bubalus bubalis) en Corrientes, Argentina, Revista electrónica de Veterinaria, 15 (1), 21-25 (2004).

Pirkul, T., A. Temiz y Y. Kernal, Fortification of yogurth with calcium salts and its effects on started microorganisms and yoghurt quality, International Dairy Journal, 7 (8-9), 547-552 (1997).

Saxelin, M., R. Korpela y A. Mäyrä-Makiren, Introduction: classifying functional dairy products, in Functional Dairy Products, CRC Press, pp 1-16, Cambridge, Inglaterra (2003).

Scholz, M. y L. Antunes, Aproveitamento da mistura de leite de vaca e de búfala para a produção de iogurte i aspectos físico-químicos e microbiológicos, Revista do ILCT, 50: 30-46 (1996).

Shojia, A.S. y otros 7 autores, Viability of L. acidophilus microcapsules and their application to buffalo milk yoghurt, Food and Bioproducts Processing, en prensa (2012).

Tamime, A. y Robinson R, Yogurt Ciencia y Tecnología, 368. Acribia, Zaragoza, España (1991).

Vélez, J. y A. Rivas, Propiedades y características del yogur. Información Tecnologica, 12 (6), 35-42 (2001).

Verruma, M. y J. Salgado, Avaliação nutricional do leite de búfala em comparação ao leite de vaca, Scientia Agricola, 51(1), 131-137 (1994). 